\title{
Planeación participativa como una alternativa de desarrollo
}

\section{The participative planning as a development alternative}

Fecha de Recepción: 3 de agosto de 2013

Fecha de Aceptación: 15 de noviembre de 2013

\author{
Helena María Barrera ${ }^{1}$, Sergio Pacheco ${ }^{1}$
}

\begin{abstract}
Resumen
Es un deber y un derecho de la comunidad y, en particular, de los profesionales, contribuir en el diseño de estrategias de equidad para el desarrollo y crecimiento de los territorios, mediante el proceso de planeación participativa, pues es allí donde se expresan las necesidades reales por las cuales se está atravesando. En este artículo se revisan los medios de participación ciudadana, se describen las relaciones de poder que se presentan en esta y se aborda la manera cómo ella promueve el desarrollo local y endógeno de los territorios, con el fin de estimular su crecimiento.
\end{abstract}

Palabras clave: Desarrollo territorial, planeación ciudadana, planeación participativa (Fuente: USDA)

\begin{abstract}
It is a duty and a community right, particularly for the professionals, to contribute to the fairness strategies' design, to the development and the territories growth, through a participatory planning process, because this is where real needs are expressed. In this article we review the citizenry participation's means, and the way it contributes to the territories development, as well as the power relations description, which arise from the participation mechanisms, and finally it considers how citizen participation promotes the local endogenous territories development, in order to stimulate their growth.
\end{abstract}

Key words: Territorial development, citizenry participation, participatory planning (Source: USDA)

1 Médicos Veterinarios Zootecnistas. Maestría en Desarrollo Rural. UPTC. helena. mvz@hotmail.es; serarpaes@gmail.com 


\section{Introducción}

La participación se da en escenarios domésticos, comunitarios, económicos, y políticos; cada uno, desde su perspectiva, le aporta al desarrollo local, que debe ser integral; al considerar equitativamente todas las dimensiones, como son: territorio, infraestructura, economía, sociedad, política y cultura (1). Así, la participación es una estrategia para lograr el desarrollo de los territorios, pues el acto de participar es mucho más que formar parte de una estructura de una cultura, es un derecho de todo ciudadano, pues lo hace partícipe en la búsqueda de soluciones a todas y cada una de las problemáticas que impiden el crecimiento de su entorno. La participación ciudadana propugna un cambio de actitudes cotidianas y, por tanto, de mentalidad; además, brinda la oportunidad de comprender lo global mediante la implicación local, y mediante la obtención precisa de las realidades se obtiene el desarrollo endógeno. En la participación, los sujetos se preparan para el cambio de actitud ante la realidad en que viven, asumiendo conductas de disciplina, responsabilidad y compromiso en la construcción de los planes de desarrollo, a los procesos de participación, por diversas razones, entre ellas la indiferencia de la ciudadanía y los factores de tiempo, que cercenan la oportunidad de exponer las necesidades de su entorno, y de plantear soluciones viables de acuerdo con las condiciones del territorio. En esas condiciones, se delega la responsabilidad a los gestores públicos, acrecentándoles la falsa idea de que ellos tienen la última palabra, y otorgándoles la autonomía para que manipulen la información y aporten soluciones de acuerdo con sus intereses personales (2).

La participación se ha trabajado como un aporte para viabilizar el desarrollo desde una construcción social, a pesar de que en la población no siempre se satisfacen las expectativas; sin embargo, en este proceso participativo surgen una serie de tipologías de participación que impacta de diferente forma el desarrollo, dentro de ellas se tienen: la destructora, la perturbadora, la funcional, la neutral y la sinérgica. Dichas tipologías de participación son útiles para orientar y contribuir a procesos de desarrollo (3).

El objeto central de este artículo es profundizar conceptualmente el alcance de la planeación participativa en diversos ámbitos académicos, sociales y políticos, que giran en torno al desarrollo de los territorios.

\section{La participación ciudadana y el desarrollo territorial}

Los ciudadanos son sujetos en los procesos de participación, pues, son un elemento importante tanto en la construcción de cambios como en el mejoramiento de las condiciones de desarrollo, con base en la organización territorial. La participación surge a partir de la Constitución Política de 1991, y de la Ley 134 de 1994, sobre los mecanismos de participación ciudadana, cuyo objetivo principal es establecer las normas fundamentales que rigen la participación democrática de las organizaciones civiles. Además, la Ley 134 de 1994 garantiza el desarrollo de otras formas de participación ciudadana en la vida política, económica, social, cultural, universitaria, sindical o gremial del país; dicha legislación pretende constitucionalizar, proteger y fortalecer la participación ciudadana a través de mecanismos como el referendo, la consulta popular, el cabildo abierto, la iniciativa legislativa y la revocatoria del mandato, y de instancias de vigilancia y control a la gestión pública, con el fin de instituir la soberanía popular y la democracia participativa (4).

El desarrollo gira en torno de la búsqueda de la calidad de vida de la población, siempre y cuando se defina el potencial de los territorios, teniendo como referencia el ordenamiento territorial, definido por la ley que lo rige (5) como:

un instrumento de planificación y de gestión de las entidades territoriales y un proceso de construcción colectiva de país, que se da de manera progresiva, gradual y flexible, con responsabilidad fiscal, tendiente a lograr una adecuada organización político-administrativa del Estado en el territorio, para facilitar el desarrollo institucional, el fortalecimiento de la identidad cultural y el desarrollo territorial, entendido este como desarrollo económicamente competitivo, socialmente justo, ambientalmente $y$ fiscalmente sostenible, regionalmente armónico, culturalmente pertinente, 
atendiendo a la diversidad cultural y físico-geográfica de Colombia.

Para Huertas (2011), la base de la organización territorial es la planificación técnica, económica, social y política, siendo necesario contar con la participación de la comunidad, generándose el medio propicio para la elaboración de los planes de desarrollo, considerados como la herramienta de gestión para las acciones de los gobernantes y habitantes de un territorio. Por otra parte, el PNUD (s.f.) considera que Colombia ha avanzado favorablemente en la elaboración de los planes de desarrollo, pues se ha mejorado la calidad y disponibilidad de información tanto a nivel nacional como territorial, para así atender las necesidades insatisfechas de la población, contribuir al desarrollo territorial y mejorar la calidad de vida de todos los ciudadanos (7).

La planeación y el diseño participativos cumplen un rol importante en la consolidación del desarrollo territorial; desde la promulgación de la Constitución de 1991 la planeación participativa ha tenido la intención de complementar la democracia de participación, reforzando la democracia representativa local y departamental (8); para Guzmán (2011), la democracia participativa se ha convertido en un lugar común que justifica sueños, como: el derecho al voto para elección de alcaldes, líderes estudiantiles, elevar un derecho de petición a una autoridad administrativa, entre otros.

Tal y como lo expresa Argáiz (s.f.), hubo un cambio drástico después de la constitucional de 1991, pues antes, la planeación era centralizada, es decir, del centro hacia la periferia, impositiva y muy técnica, ya que no se tenía en cuenta el conocimiento existente, pues el Estado homogenizaba las necesidades, debido a que ignoraba las necesidades reales de los diversos sectores, y no se promovían el diálogo ni el consenso; sin embargo, Bustamante (2011) indica que los avances para superar el autoritarismo, la exclusión y los vicios políticos del sistema político y social son escasos; a pesar de la buena intención de la Constituyente, en Colombia no se ha fortalecido la democracia; sin embargo, a partir de la Constitución de 1991 las relaciones Estado - Individuo son más accesibles, ya que la democracia participativa permite a los ciudadanos acercarse más a sus representantes, no como observadores, sino como sujetos activos en la toma de decisiones (11).

En este contexto, Velásquez (2013) indica que la democracia participativa transformó la democracia representativa, modelo clásico de participación política, asociada al surgimiento del EstadoNación moderno, por razones de diferente índole, como: el tamaño de los territorios, la imposibilidad de participación de todos los ciudadanos y; las restricciones a la participación, asociados a mecanismos como el denominado voto censitario. Todo lo anterior dio lugar a que en la Constitucional de 1991 se modificaran las formas de participación y se estableciera un rango constitucional para organismos de participación ciudadana, como el Consejo Nacional de Planeación.

De otro lado, la ley orgánica 152 de 1994 consolidó uno de los esquemas básicos, institucionales y de procedimiento para la ejecución de la planeación participativa, considerando una variedad de instrumentos de planeación, tales como: los planes de desarrollo, de ordenamiento territorial, los planes maestros, los planes sectoriales, las políticas públicas poblacionales y sectoriales, entre otros (13).

Sin embargo, González y Velásquez (2010) indican que se han presentado fallas que han estimulado ciertas reformas, tales como la introducción de los principios de diversidad, inclusión, territorialidad, equidad de género y enfoque de derechos; también indican que el Sistema Nacional de Planeación tiene la necesidad de contar con una reglamentación que defina sus funciones, su estructura básica, las reglas para la conformación de sus instancias, el tipo de actividades que puede y debe desarrollar, los instrumentos a su alcance para el desempeño de sus funciones y sus interlocutores en el ámbito del Estado.

\section{Las relaciones de poder en la participación}

El medio para alcanzar metas, logros, proyectos de beneficio común es la participación, a partir de la cual se inician las relaciones de poder, permitiendo que estas se difundan (Bustamante, 2011). La participación es asociada a la libertad de las personas, quienes pueden otorgar poder frente 
a otros poderes existentes en nuestras sociedades, como en los casos de los partidos políticos, las organizaciones gremiales y los medios de comunicación, entre otros.

Según Villarreal (1999), el poder es central en las relaciones entre hombres y mujeres, y define la forma y contenido de estas; sin embargo, aún se presentan inequidades, pues aunque se plantea que quienes participan en las relaciones de poder no carecen totalmente de poder, no se desglosa cuáles son los poderes con que cuentan las mujeres; más bien, se profundiza en sus roles como subordinadas y se cae casi en una victimización, lo cual impide visualizar sus poderes -aunque limitados- para replantear dichas relaciones. Por otro lado, el poder en un proceso de cambio organizacional es la variable que interviene entre los resultados deseados y los obtenidos; sin embargo, se advirtió que las distintas bases de poder ejercen de manera diferente su potencial de influencia, interviniendo en mayor o menor grado en los resultados esperados (16).

De otro lado, algunas relaciones de poder vulneran y atentan contra la cultura propia, y se adquiere una impuesta y enajenada, dejando de lado el etnodesarrollo, entendido como el ejercicio de la capacidad social de un pueblo para construir el futuro (17), aprovechando para ello las enseñanzas de su experiencia histórica y los recursos reales y potenciales de su cultura, de acuerdo con un proyecto que se defina según sus propios valores y aspiraciones. Sin embargo, en las últimas décadas, frente a la crisis del modelo económico global, se han hecho nuevos planteamientos que propician una mayor participación democrática, a través de medidas incluyentes fundamentadas en la libertad y la diversidad cultural y étnica, puesto que dentro del marco de poder político el etnodesarrollo busca tener la oportunidad de participar en las decisiones con carácter autónomo o de cultura propia. Además, el etnodesarrollo sugiere un cambio de la correlación de fuerzas sociales, en un campo político que favorezca a los que han sido desconocidos en la práctica de las relaciones culturales e históricas, a las cuales se les reconoce un territorio dentro de la nación (18).

\section{El desarrollo local y endógeno}

El desarrollo local promueve la participación y el diálogo a nivel local, puesto que todos los actores sociales, públicos o privados, así como sus recursos son considerados elementos importantes; luego deben ser movilizados para participar y trabajar en este modelo de desarrollo, con miras a lograr una mejor calidad de vida de la población (19). El desarrollo local requiere recursos financieros y e información; los primeros permiten la contratación de entidades y de organismos encargados del servicio a la comunidad, mientras que el segundo permite generar influencia social respecto a la visión que se tenga del mundo; por lo anterior el principal responsable para que esto se dé es el Estado Colombiano (20).

Por otra parte, el desarrollo endógeno considera importante factores, como: el bienestar material, social y espiritual, de tal forma que logre la satisfacción de las necesidades básicas, la participación de la comunidad, la protección del ambiente y la localización de la comunidad en un espacio determinado (21). La propuesta del desarrollo endógeno de las sociedades contemporáneas es la participación efectiva, y el respeto de los derechos humanos, pues de ellos depende el progreso duradero, a través del planeamiento participativo, como instrumento fundamental para orientar el futuro analizando la evolución del enfoque de su planificación (22).

Según lo anterior, para lograr el desarrollo territorial se requiere, necesariamente, lograr el desarrollo local y endógeno, puesto que el desarrollo es un proceso que aborda el conjunto de problemas sociales, económicos y medioambientales de un territorio y determina soluciones integradas para la creación de empleo y el trabajo decente (CEIF). Además, el desarrollo es un modelo socioeconómico en el que las comunidades realizan sus propias propuestas; la meta de este modelo es generar el desarrollo a nivel local, de la comunidad, pero que trascienda hacia arriba, hacia la economía del país, hacia el mundo, en otras palabras, de lo particular a lo general (23). Harnecker y López (2009) afirman que el grado de compromiso que adquieren las personas en los proceso de planificación es alto, pues se busca 
cumplir las metas propuestas por la comunidad, y no las que se plantean desde la periferia, que en la mayoría de los casos son promesas que no se cumplen.

\section{Conclusión}

De acuerdo con Echeverry (2010), la oportunidad que le dio la Constitución de 1991 a la participación ciudadana, y su evidente trascendencia, ha sido de muy buena aceptación por los ciudadanos; sin embargo, en vigencia de las leyes mencionadas y otras afines, han surgido numerosas críticas con respecto a la efectividad de los mecanismos de participación y control social, ya sea porque el Estado no los apoya o porque al constatar su escasa aplicación, la ciudadanía no los usa adecuadamente o no les confiere la suficiente importancia; en consecuencia, se ha venido generando un desconcierto, que a su vez ha agudizado la indiferencia ciudadana.

Por otra parte, algunas dificultades por las que se frustran los procesos de participación están relacionadas con el grado de comprensión que el hombre de la calle pueda tener sobre ciertos temas que lo afecten, pero al no entenderlos no opina sobre ellos; además, es evidente que buena parte de la población hace uso de su derecho de participación, mientras que el número de asuntos vitales para la comunidad, que requieren decisión por alguna dependencia del Estado, crece aceleradamente, y el conocimiento de cada una de las necesidades requiere más consultas populares y actividad, lo cual se torna algo dispendioso y poco práctico (26).

Una de las grandes dificultades de la participación es el divorcio existente entre los esquemas de representación política y los de la participación ciudadana; en Colombia, la participación fue entendida desde un comienzo como un sustituto, no como un complemento, de la representación política; ese fue el lenguaje dominante, tanto en el seno de los partidos políticos como de la dirigencia social. Las desconfianzas y las descalificaciones mutuas han sido pan de cada día: los políticos, aduciendo la baja representatividad y la poca legitimidad de los actores de la participación; los líderes sociales, satanizando el ejercicio de la política, al calificarla como nido de corrupción y de falsas promesas; los partidos, desinteresándose de la participación; los actores de la participación, calificando la influencia partidista como contaminante de la supuesta "pureza" del mundo social (27).

Por otra parte, y de acuerdo con Zuluaga et al. (2012), el respeto por los recursos naturales, la construcción de ciudadanía, el fortalecimiento de identidades y el sentido de pertenencia hacia el territorio, concebido como un sistema dinámico, en proceso de estructuración y permanente evolución, son las bases sobre las cuales deben girar las políticas públicas de desarrollo de los territorios regionales de tal manera que se garantice el desarrollo humano con igualdad social, bienestar y calidad de vida para las presentes y futuras generaciones.

Finalmente, aunque el camino de la participación existe, no será fácil recorrerlo y lograrlo; dependerá, en últimas, de la ciudadanía, cuando logre superar los obstáculos mentales, materiales, y legales que aún impiden usar adecuada y efectivamente la participación ciudadana en Colombia.

\section{Referencias bibliográficas}

(1) Barroso C. (s.f.). Economía social, Participación y Desarrollo Local. Red canaria rural (en línea) 2008 (fecha de acceso 13 de septiembre de 2013). URL disponible en: http:// www. Redcanariarural.org/pdf/06_barroso_ribal.pdf

(2) Perero E. (s.f.). Participación Ciudadana. CONAMA (en línea) 2009 (fecha de acceso 14 de septiembre de 2013). URL disponible en: http://www.conama.es/viconama/ds/ pdf/52.pdf

(3) Múnera MC, Sánchez LM. La participación en la sociedad como base del desarrollo: Aproximación a tipologías de participación. UNAL (en línea) 2008 (fecha de acceso 18 de septiembre de 2013). URL disponible en: http://www.bdigital.unal.edu.co/3356/1/ MCM-LILI-ACIUR.pdf 
(4) Muñoz F. (s.f.). Análisis Ley de Participación. MOE.org (en línea) 2007 (fecha de acceso 27 de agosto de 2013). URL disponible en: http:// moe.org.co/reformas-politicas-y-electorales/ estatuto-participacion-ciudadana/451-analisisley-de-participacion.html

(5) Congreso de Colombia. Ley 1454 de 2011. Por la cual se dictan normas orgánicas sobre ordenamiento territorial y se modifican otras disposiciones (en línea) 2011 (fecha de acceso 12 de agosto de 2013). URL disponible en: http://www.bibliotecajuridica.com.co/ LEY_1454_DE_2011.pdf

(6) Huertas E. El Diseño y la Planeación Técnica Participante: Instrumento para la Administración Democrática del Territorio Municipal. UNAL (en línea) 2011 (fecha de acceso 23 de agosto de 2013). URL disponible en: http://www.bdigital.unal.edu.co/3613/1/ EHG-PlanParticipRevForo.pdf

(7) Argáiz A. (s.f.). Coordinación Política. PNUD (en línea) 2009 (fecha de acceso 14 de Agosto de 2013). URL disponible en: http: //www.sre. gob.mx/coordinacionpolitica/images/stories/ documentos_gobiernos/pmpnud6.pdf

(8) Forero C. El Sistema Nacional de Planeación Participativa en Colombia 1994- 2000. ECLAC (en línea) 2000 (fecha de acceso 15 de Agosto de 2013). URL disponible en: http://www. eclac.org. Colombia_PlaneacionParticipativa

(9) Guzmán A. Democracia Participativa en Colombia: Un Sueño Veinte Años Después. UCALDAS (en línea) 2011 (fecha de consulta 14 de agosto de 2013). URL disponible en: http://juridicas.ucaldas.edu.co/downloads/ Juridicas8(2)_2.pdf

(10) Bustamante G. Qué pasó con la Democracia Participativa en Colombia. Rev Semana (en línea) 2011 (fecha de acceso 10 de agosto de 2013). URL disponible en: http://www. semana.com/nación/articulo/que-pasodemocracia-participativa/238906-3

(11) Moisés C. Cabildeo y su Incidencia en la Democracia Participativa. PUJ (en línea) 2011 (fecha de acceso: 14 de agosto de 2013). URL disponible en: http:// www.javeriana.edu.co/ biblos/tesis/derecho/dere2/Tesis45.pdf

(12) Velásquez A. Democracia Representativa y Democracia Participativa. El Colombiano (en línea) 2013 (fecha de acceso: 20 de agosto de 2013). URL disponible en:http://www. elcolombiano.com/BancoConocimiento/D/ democracia_representativa_ydemocracia participativa/democracia_representativa_y_ democracia_participativa.Āsp

(13) Congreso de Colombia. Ley 152 de 1994 (en línea) 1994 (fecha de acceso 12 de agosto de 2013). URL disponible en: http://www. secretariasenado.gov.co/senado/basedoc/ ley/1994/ley_0152_1994.html

(14) González E, Velásquez F. La Planeación Participativa, el Sistema Nacional de Planeación y los Presupuestos Participativos en Colombia. VIVA org (en línea) 2010 (fecha de acceso 16 de agosto de 2013). URL disponible en: http://www.viva.org.co/ documentos/cat_view/4-red-nacional-deplaneacion-local-y-presupuesto-participativo

(15) Villarreal A. Relaciones de Poder en la Sociedad Patriarcal. Rev INIE (en línea) 1999 (fecha de acceso 20 de agosto de 2013). URL disponible en: http://revista.inie.ucr.ac.cr/ uploads/tx_magazine/patriarcal.pdf

(16) Caicedo S, Córdoba M, González C. Análisis de las Relaciones de Poder en un Proceso de Cambio Organizacional Planificado. PUJ (en línea) 1996 (fecha de acceso 18 de agosto de 2013) URL disponible en: http://pujportal.javeriana.edu.co/portal/page/portal/ Facultad\%20Psicologia/recursos/documento_ analisis_relaciones.pdf

(17) Bonfil G. Etnodesarrollo: sus premisas jurídicas, políticas y de organización. Udvirtual (en línea) 1995 (fecha de acceso 19 de agosto de 2013). URL disponible en: http://www.udgvirtual.udg.mx/dspace/ bitstream/20050101/1269/2/etnodesarrollo. pdf 
(18) Mosquera O. (s.f.). El Etnodesarrollo: Un Camino para la Inclusión Social. Convergenciacnoa (en línea) 2008 (fecha de acceso 20 de agosto de 2013). URL disponible en: http://www.convergenciacnoa.org/files/ El\%20etnodesarrollo $\%$ 20un $\% 20$ camino $\%$ 20para $\% 20$ la $\% \quad$ 20inclusi $\%$ C3 $\%$ B3n $\% 20$ social.pdf

(19) Couto S. Qué es el Desarrollo Local (en línea) 2006 (fecha de acceso 18 de agosto de 2013). URL disponible en: http://www.unilibrecali. edu.co/programa-economia/\% 5Cimages \% 5Cstories\%5Cforma_altonivel\%5C_es_DL_ Couto_Sueli.pdf

(20) Brand E. Redes interinstitucionales en la planeación participativa para el desarrollo local. El Análisis de Redes Sociales en el marco de la Gobernanza Moderna (en línea) 2008 (fecha de acceso 30 de septiembre de 2013). URL Disponible en: http:// www. analisisredes.com.ar/htm/Redes $\% 20$ interinstitucionales $\% 20$ en $\% 201$ a $\% 20$ planeacion $\% \quad$ 20participativa $\% 20$ para $\% 20$ el\%20desarrollo\%20local.htm

(21) Boonzaaijer C. Desarrollo Endógeno. Rev COMPAS (en línea) 2008 (fecha de acceso 14 de agosto de 2013). URL disponible en: http:// www.agruco.org/compas/pdf/ COMPAS $\% 20$ 13.pdf

(22) Tauber F. El desarrollo y su planificación. Evolución del concepto y su influencia en procesos urbanos endógenos, sustentables y participativos. UPC (en línea) 2012 (fecha de acceso 1 de septiembre de 2013). URL disponible en: https://upcommons.upc. edu/revistes/bitstream/2099/12501/1/05_0_ FernandoTauber.pdf

(23) PSUV Partido Socialista Unido de Venezuela. Desarrollo Endógeno. Desde adentro, desde la
Venezuela profunda (en línea) 2008 (fecha de acceso 15 de agosto de 2013). URL disponible en: http://aristobulo.psuv.org.ve/wp-content/ uploads/2008/10/desarrollo_endógeno_2.pdf

(24) Harnecker M, López N. Planificación Participativa en la Comunidad. Rebelión (en línea) 2009 (fecha de acceso 15 de agosto de 2013). URL disponible en: http://www. rebelion.org/docs/97084.pdf

(25) Echeverry CF. La Participación Ciudadana en Colombia: Reflexiones desde la Perspectiva Constitucional y la Normatividad Estatuaria (en línea) 2010 (fecha de acceso 28 de agosto de 2013). URL disponible en: http:// aprendeenlinea.udea.edu.co/revistas/index. php/red/article/viewFile/8628/7963

(26) Echeverri F. El fin de la Democracia Participativa. El Tiempo (en línea) 1991 (fecha de acceso 20 de agosto de 2013). URL disponible en: http://www.Eltiempo.com/ archivo/documento/MAM-25907

(27) Velásquez F. Una Nueva Ley Estatutaria de Participación. Razón Pública.com (en línea) 2011 (fecha de acceso 29 de agosto de 2013). URL disponible en http://www.razonpublica. com/index.php/recomendado/2359-unanueva-ley-estatutaria-de-participacion.html

(28) Zuluaga ML, Mosquera J, Gómez RE, Peñalosa FJ. Construcción Colectiva de Políticas Públicas para el Desarrollo Humano Sostenible. Rev Lunazul (en línea) 2012 (fecha de acceso 15 de septiembre de 2013). URL disponible en: http://lunazul.ucaldas.edu.co/index. php? option $=$ content $\&$ task $=$ view $\&$ id $=744$ 
